\title{
A aplicação de um guia de medidas preventivas por médicos de família em um centro de saúde de Ribeirão Preto, São Paulo, Brasil
}

\author{
The application of a guide for disease prevention by family physicians in a health centre of \\ Ribeirão Preto, São Paulo, Brazil
}

\author{
La aplicación de un guía de medidas preventivas por los médicos de familia en un centro de \\ salud de Ribeirão Preto, São Paulo, Brasil
}

Luciane Loures dos Santos. Universidade de São Paulo (USP). Ribeirão Preto, SP, Brasil. Iuloures@hotmail.com (Autora correspondente)

Anderson Soares da Silva. Universidade de São Paulo (USP). Ribeirão Preto, SP, Brasil. assilva@fmrp.usp.br

Amaury Lelis Dal-Fabbro. Universidade de São Paulo (USP). Ribeirão Preto, SP, Brasil. adfabbro@fmrp.usp.br

Luciana Martins Versiani. Secretaria de Estado de Saúde do Distrito Federal (SES-DF). Brasília, DF, Brasil. luversiani@gmail.com

Jose Sebastião dos Santos. Universidade de São Paulo (USP). Ribeirão Preto, SP, Brasil. jsdsanto@fmrp.usp.br

Ajith Kumar Sankarankutty. Universidade de São Paulo (USP). Ribeirão Preto, SP, Brasil. ajith@fmrp.usp.br

\section{Resumo}

Neste estudo, foi adaptado um protocolo de prevenção, contendo medidas de prevenção primária e secundária, composto por rastreamento, aconselhamento, vacinação e quimioprofilaxia com níveis A e B de recomendação. 0 estudo foi desenvolvido em uma Unidade de Saúde da Família na qual atuam residentes de Medicina de Família e Comunidade. Os médicos foram treinados quanto à importância e utilização do material preventivo (tabela e encarte-lembrete) e sua adesão foi avaliada por meio do preenchimento do encarte-lembrete. No ano estudado, realizaram-se 3.995 consultas a 1.596 usuários e, em apenas 397 prontuários, encontrou-se o encarte-lembrete com registro de uma medida preventiva, o que representou 25\% da população atendida. As medidas encontradas seguiram esta ordem: rastreamento (99\%); aconselhamento (90\%); quimioprofilaxia (55\%); e vacinação (45\%). Este protocolo de prevenção norteia o profissional quanto às medidas a serem oferecidas, entretanto faz-se necessário implementar outras ações para que as medidas preventivas possam ser oferecidas de maneira mais efetiva.

\section{Abstract}

In this study, we adapted a protocol for preventive measures containing primary and secondary prevention, comprising screening, counseling, vaccination and chemoprophylaxis with levels A and B of recommendation. This study was conducted in a Family Healthcare Unit where Family and Community Medicine residents are trained. Medical resident students were trained on the importance and use of prevention material (protocol and reminders) and their adherence was assessed by the completion of booklet reminders. During the one-year study period, 3995 consultations were held with 1596 patients. However, the record of at least one preventive measure was found in only 397 booklet reminders, which represents $25 \%$ of the population seen. The preventive measures found were screening $(99 \%)$, counseling $(90 \%)$, chemoprophylaxis $(55 \%)$, and vaccination $(45 \%)$. This protocol provides professionals with a set of preventive measures to be offered; however, other actions are needed so that preventive measures can be provided more effectively.

\section{Resumen}

En este estudio, fué adaptado un un protocolo de medidas de prevención primarias y secundarias, compuesto por selección, consejo, vacunación y quimioprofilaxis con los niveles A y B de recomendación. El estudio se realizó en una Unidad de Salud Familiar, donde trabajan residentes de Medicina Familiar y Comunitaria. Se capacitó a los médicos sobre la importancia y el uso del material de prevención (tabla y folleto recordatorio) y se evaluó su adhesión mediante las respuestas al cuestionario localizado en el folleto recordatorio. En el año de estudio, los médicos realizaron 3995 consultas a 1596 pacientes y en sólo 397 historias clínicas se encontró el folleto recordatorio con registro de una medida preventiva, lo que representa el 25\% de la población atendida. Las medidas encontradas siguieron este orden: selección (99\%), consejo (90\%), quimioprofilaxis (55\%) y vacunación (45\%). Este protocolo de prevención guía a los profesionales sobre las medidas que se deben ofrecer, sin embargo, es necesario implementar otras acciones para proporcionar las medidas preventivas con mayor efectividad.
Palavras-chave: Prevenção de Doenças Saúde da Família Avaliação de Resultado de Ações Preventivas

\section{Keywords:}

Disease Prevention Family Health Evaluation of Results of Preventive Actions

\section{Palabras clave:} Prevención de Enfermedades Salud de la Familia Evaluación de los Resultados de Acciones Preventivas

\section{Parecer CEP:}

of.n. ${ }^{\circ}$ 031/08/COORD.CEP/

CSE-FMRP-USP. 28/02/2008.

Fonte de financiamento:

Fundação Waldemar Barnsley Pessoa.

Conflito de interesses: declaram não haver. Recebido em: 07/05/2012 Aprovado em: 24/05/2013 


\section{Introdução}

As doenças crônicas não transmissíveis (DCNT) estão entre as principais causas de morte no mundo, sendo que $80 \%$ ocorrem nos países de baixa e média renda ${ }^{1}$. A maioria desses óbitos é causada por doenças do aparelho circulatório, câncer, diabetes e doenças respiratórias crônicas, cujos principais fatores de risco são o tabagismo, o alcoolismo, o sedentarismo e a alimentação não saudável ${ }^{2}$. Uma vez que se conhecem os fatores de risco, a próxima etapa seria combatê-los ou controlá-los. Cientes disso, faz-se necessário reorganizar as prioridades dos cuidados em saúde, que atualmente tem ênfase no modelo curativo, para uma forma de cuidar que também produza promoçáo da saúde e prevenção de doenças (PSPD), exercendo a integralidade da atenção, nem só cura e tratamento, nem tampouco só prevenção ${ }^{3,4}$.

Alguns países industrializados e de alta renda trabalham com protocolos de prevenção clínica, elaborados por órgãos governamentais, baseados em evidências científicas (que envolvem prevenção primária e secundária) por meio de módulos de rastreamento, aconselhamento, vacinação e quimioprofilaxia. Tal abordagem faz parte do que se convencionou chamar "Exame Periódico de Saúde" (EPS) e tem como objetivo prevenir o aparecimento de doenças, identificar estados patológicos em estágios assintomáticos e melhorar o estado de saúde do indivíduo e da comunidade 5 .

Em 2005, no Brasil, não existiam protocolos ou propostas centradas nos indivíduos, que levassem em conta o gênero, a faixa etária, e fatores de risco individuais, adaptados à situação epidemiológica do País, nem a aplicação por profissionais da atenção primária. Por isso surgiu a necessidade de se adaptar um protocolo com tais características para ser aplicado no serviço. Nessa linha, apenas em 2010, o Ministério da Saúde lançou o Caderno de Atenção Básica, número 29, denominado de "Rastreamento"6.

\section{Contextualização}

O estudo foi realizado em uma Unidade de Saúde da Família (USF) no distrito oeste do município de Ribeirão Preto, totalmente servida por saneamento básico, energia elétrica, abastecimento de água por rede pública e lixo coletado. A equipe de saúde da família era composta por um médico de família e comunidade, um enfermeiro, dois auxiliares de enfermagem, um auxiliar de serviços gerais e cinco agentes comunitários de saúde responsáveis pelo atendimento de 902 famílias cadastradas, totalizando 3.120 pessoas. Destas, 1.704 eram mulheres (55\%) e 1.416 eram do sexo masculino (45\%), com predomínio de adultos jovens entre 20 e 39 anos $(30,2 \%)$ e idosos (22,1\%) com maior prevalência de doenças crônico-degenerativas.

Além de prestar assistência clínica aos munícipes, esta unidade serve de campo de ensino e pesquisa a alunos de graduação e pós-graduaçáo da universidade, além de treinamento de médicos residentes do Programa de Residência em Medicina de Família e Comunidade (MFC).

No período estudado estagiavam ali, cinco residentes de MFC, sendo dois residentes do segundo ano e três residentes do primeiro ano. Cada residente era responsável pela assistência e gestão de uma microárea e todas as açóes eram discutidas com o médico de família responsável pela USF.

\section{Descrição}

\section{Elaboração do material empregado}

Foi elaborado um protocolo de prevenção clínica contendo 22 medidas de prevenção primária e secundária, denominado "O Exame Periódico no Universo da Atenção Primária", tendo como base recomendaçóes de instituiçóes nacionais e internacionais, adaptadas às necessidades locais ${ }^{7}$. Esse protocolo de prevenção foi composto por medidas de rastreamento, aconselhamento, vacinação e quimioprofilaxia, classificadas como níveis de evidência A e B de recomendação ${ }^{8}$, selecionadas segundo as diretrizes do U.S. Preventive Services Task Force ${ }^{9}$, do Canadian Task Force on Preventive Health Care ${ }^{10}$, do Programa de Actividades Preventivas y de Promoción de la Salud (PAPPS) ${ }^{11}$ da Sociedade Espanhola de Medicina de Família e Comunidade 
(SemFYC), além de recomendaçôes de prevenção em saúde do Ministério da Saúde e da Secretaria Municipal da Saúde de Ribeirão Preto, São Paulo.

Preocupados com os custos e com a viabilidade de oferecer um rastreamento com os quais os serviços locais pudessem arcar, foram utilizados apenas os parâmetros plenamente aceitos. Desta forma, considerou-se este conjunto de medidas como 'o mínimo necessário a ser oferecido à população' baseado no conhecimento médico científico disponível naquele momento. A partir deste protocolo e de acordo com a população cadastrada no Sistema de Informação da Atenção Básica (SIAB), estimaram-se os números de encontros e exames complementares esperados, assim foi possível fazer uma previsão das necessidades locais.

Para facilitar a aplicação do protocolo, foi proposta uma tabela com o resumo do EPS, orientando o profissional quanto ao que deveria ser investigado, segundo idade, sexo e a presença ou não de fator de risco e com qual periodicidade cada medida precisaria ser empregada (Anexo 1). Essa tabela estava disponível aos profissionais de saúde nos consultórios da unidade em que trabalhavam ou estagiavam.

Também, foi elaborado um encarte-lembrete em que deveriam ser registradas as medidas aplicadas, as que estavam pendentes, as que estavam normais e as alteradas (Anexo 2). Os profissionais de saúde foram orientados a afixar o encarte-lembrete como folha de rosto do prontuário clínico individual, devendo atualizá-lo à medida que as açôes de prevenção fossem sendo empregadas.

\section{Treinamento}

Todos os profissionais da equipe de Saúde da Família foram sensibilizados quanto à relevância da prevenção no âmbito da atençáo primária, assim como sobre os benefícios das medidas preventivas. Os profissionais foram orientados a oferecer as medidas preventivas de maneira sistemática a todos os usuários que buscassem a USF, tanto por meio de consultas agendadas, consultas eventuais, grupos ou durante as visitas domiciliares. Ademais, as 22 medidas presentes no protocolo de prevenção, foram discutidas, assim como a utilização da tabela resumo e o registro no encarte-lembrete das medidas de prevenção aplicadas. Ainda como parte do treinamento, os residentes de MFC participaram de simulaçóes a partir de casos clínicos, para aperfeiçoar o uso das medidas preventivas na prática clínica.

Maior ênfase foi dada a equipe médica, composta por um médico de família e comunidade e cinco médicos residentes de MFC (sendo dois residentes do segundo ano e três do primeiro ano), que participaram de todo o processo de implantação do EPS segundo as diretrizes contidas no protocolo "O Exame Periódico no Universo da Atenção Primária".

A adesão dos profissionais ao uso do protocolo de prevenção foi verificada por meio do preenchimento dos encartes-lembrete afixados aos prontuários individuais. Para tanto, todos os prontuários clínicos da unidade foram revisados, buscando-se aqueles cujos usuários tiveram pelo menos uma consulta médica durante o ano de 2005, pois, neste estudo, foi analisada apenas a aplicação das medidas preventivas por médicos de família. Foram excluídos os prontuários das gestantes, dos usuários que mudaram de área ou que faleceram. Também foram excluídos os atendimentos dos usuários de fora da área, pois, normalmente, são realizadas consultas pontuais de usuários que não moram na área adstrita.

Como o protocolo de prevençấo foi desenvolvido para maiores de 11 anos e a faixa etária do SIAB inclui as idades de 10 a 14 anos, optou-se também por excluir os prontuários dos menores de 14 anos.

\section{Resultados}

Os usuários que mais procuraram atendimento médico na unidade foram os maiores de 60 anos (35\%) e aqueles com idade entre 20 e 39 anos (25\%), seguidos daqueles entre 50 e 59 anos (17\%) e 40 e 49 anos (16\%).

Foram realizadas 4.701 consultas médicas no período analisado, contudo foram excluídos os menores de 14 anos e aqueles atendimentos de usuários residentes fora da área de abrangência da unidade, e analisados 3.995 atendimentos de 1.596 pacientes. Considerando apenas os atendimentos de usuários incluídos no estudo (1.596 pacientes), calculou-se a média de 2,5 consultas por paciente no período. 
A revisão dos prontuários médicos dos 1.596 pacientes atendidos mostrou que apenas 397 deles continham o encarte-lembrete com o registro de ao menos uma medida de PSPD, correspondendo a $25 \%$ dos atendimentos realizados.

Quanto aos tipos de medidas de PSPD empregadas, aquelas que apresentaram maior porcentagem de registro foram: rastreamento (99\%) e o aconselhamento (90\%). A quimioprofilaxia e a vacinaçáo apresentaram um percentual mais baixo, respectivamente, $55 \%$ e $45 \%$.

\section{Discussão}

Embora as USF sejam locais privilegiados para a aplicação das medidas de PSPD, foi observada uma baixa frequência de medidas de prevenção clínica registradas nos encartes-lembrete.

Acredita-se que o treinamento não tenha sido suficiente a ponto de sensibilizar os profissionais quanto à importância do emprego das medidas preventivas. Algumas medidas preventivas podem não ter sido registradas devidamente nos prontuários. Ademais, talvez fosse necessário mudar a metodologia do treinamento, incluindo mais espaços de discussão sobre o tema entre todos os profissionais da equipe de saúde da família.

É importante lembrar que o médico assume papel central na assistência à saúde, contudo sua atuação corresponde apenas a uma parte da assistência, devendo-se considerar a participação dos demais membros da equipe da USF. O trabalho em equipe é essencial para a aplicação de medidas de prevenção na Atenção Primária à Saúde, basta considerar que no ano do estudo, segundo dados do SIAB, foram realizadas 8.677 visitas domiciliares pela equipe, sendo 7.880 visitas feitas pelos agentes comunitários de saúde, 150 pela equipe de enfermagem e 284 pela equipe médica, além das visitas de outros profissionais (psicólogos, nutricionistas, fisioterapeutas, farmacêuticos e fonoaudiólogos). Tais visitas poderiam ser aproveitadas para a aplicação de algumas medidas de prevenção, tais como, verificação da carteira de vacina, aconselhamento sobre a importância da realização de mamografias ou dos malefícios do tabagismo, entre outras e portanto, o treinamento de toda a equipe, possivelmente, ajudaria na melhoria dos índices de aplicação das medidas de prevenção. A educação em saúde, visando maior adesão da população, seria outro ponto no qual investir.

Um dos fatores que também pode ter contribuído para o baixo índice de emprego das medidas de prevenção diz respeito à própria formação dos profissionais de saúde. Sabe-se que, durante a graduação, a maioria dos cursos superiores da área é voltada para uma formação eminentemente curativa e hospitalar, ficando em segundo plano a abordagem de temas relacionados à promoção da saúde e prevenção de doenças. De fato, mudar a visão do profissional em relação às medidas preventivas requer profundas alteraçóes na maneira como eles estão sendo formados nas universidades, por exemplo, com a criação de disciplinas específicas e a inserção precoce do aluno em unidades de cuidados primários ${ }^{12}$.

Uma das limitaçôes do estudo foi a busca da informação apenas nos encartes-lembrete contidos nos prontuários. Em vários prontuários, não foi encontrado e, quando encontrado, não estava preenchido. Tal achado está de acordo com vários trabalhos nacionais, pois o registro em prontuário, seja no âmbito hospitalar ou ambulatorial, apresenta deficiências, como prontuários mal preenchidos, incompletos e com letras ilegíveis ${ }^{13-15}$. Seria necessário revisar todo o prontuário clínico individual, já que as medidas poderiam ter sido aplicadas e registradas ao longo das consultas. Ou ainda, buscar a informação em outros instrumentos utilizados pelas equipes da ESF, tais como: fichas de acolhimento, prontuário familiar e cartão de vacina.

Por ser uma USF que também trabalha com ensino e pesquisa, os autores se surpreenderam com o baixo registro encontrado nos encartes-lembrete. Acredita-se que os inúmeros locais de registro, como o prontuário familiar e individual, além dos diferentes tipos de Sistemas de Informação em Saúde que estão presentes na Atenção Primária à Saúde (SIAB, Hiperdia, Siscolo, etc.) acabam por dificultar o registro das informaçôes. Tornam-se necessários sistemas de informação que se comuniquem, facilitando o registro da assistência prestada pela equipe de saúde, produzindo dados mais fidedignos e com mais agilidade. Estudos evidenciam que a criação e utilização de recordatórios eletrônicos facilitam a aplicação e o registro das medidas preventivas ${ }^{16}$.

Ademais, as USF não podem ser os únicos locais a empregarem as medidas de prevenção clínica. Segundo os dados encontrados no SIAB, 33,6\% dos indivíduos cadastrados possuíam acesso à saúde suplementar. Além disso, 45\% deles responderam que buscavam outros serviços de saúde, além do atendimento prestado pela USF. Ressalta-se que a aplicação das medidas preventivas deve ser realizada em cada encontro com os usuários, por todos os profissionais da ESF, na unidade, no domicílio e mesmo nos equipamentos sociais. 
Tal tarefa poderia ser facilitada não apenas com a conscientização dos profissionais (por meio de treinamentos ou educação médica continuada), mas também pela utilização de protocolos eletrônicos que funcionariam como lembretes, ao incluir nos prontuários individuais e familiares da USF, recomendações de medidas de prevenção segundo o sexo, a faixa etária e os fatores de risco individuais ${ }^{17}$.

Vale ressaltar que existem inúmeros aspectos preventivos ainda não bem estabelecidos, como o intervalo para solicitação de alguns exames de rastreamento ou com qual frequência deve-se aplicar algumas medidas. Vive-se uma pressão pelo incremento da prevenção, seja por interesses na venda de mais medicamentos, inovação em aparelhos de screening, além da diminuição dos limites das pré-doenças. Neste cenário, torna-se imprescindível incluir, entre as medidas de PSPD, apenas aquelas que têm indiscutivelmente o apoio da literatura científica. Essa é uma das tarefas dos médicos da ESF, profissionais fundamentais para o sistema público de saúde, visto que $70 \%$ dos diagnósticos de câncer são realizados por médicos não oncologistas $^{18,19}$.

Os protocolos de prevenção são recomendaçôes de conduta e não "camisas de força" a engessar a prática clínica. O profissional de saúde deve exercer uma avaliação crítica da situação e distinguir elementos que fogem da rotina e, portanto, da aplicação do protocolo. É também papel da equipe da ESF realizar a prevenção quaternária, identificando usuários que possam estar expostos a intervençóes terapêuticas ou diagnósticas desnecessárias e inapropriadas ${ }^{6}$.

Por fim, as ações preventivas de abordagem individual, como o rastreamento oportunístico, direcionado a populações de alto risco, podem ser menos efetivas e mais caras que outras abordagens de âmbito populacional. No entanto, na prática clínica de uma equipe da ESF, quando se pensa na assistência centrada no usuário, é importante que este tenha acesso aos serviços de saúde e às informações sobre o cuidado com sua saúde. É preciso oferecer aos usuários as medidas preventivas indicadas para o seu perfil, para que ele tenha acesso à informaçáo e o livre arbítrio para decidir sobre a realização ou não de exames, prescriçôes ou orientaçôes ${ }^{20}$.

Os motivos econômicos podem não justificar a aplicação das medidas de PSPD no âmbito individual e oportunístico, bem como, deve-se pesar os potenciais benefícios e danos para a saúde dos indivíduos que têm um diagnóstico precoce. Por outro lado, um tratamento efetivo que diminui o sofrimento, a dor e até a morte por causas evitáveis, é um argumento real e razão para a implementação de medidas preventivas ${ }^{21}$.

\section{Conclusão}

O Exame Periódico de Saúde é um importante instrumento norteador das medidas de promoção de saúde e prevenção de doenças que devem ser oferecidas aos usuários e à populaçáo cadastrada, no entanto, disponibilizar protocolos e instrumentos que facilitem a implantaçáo das medidas de PSPD não se mostrou suficiente. Provavelmente, outras medidas precisam ser implementadas na atenção primária, para que as medidas de PSPD possam ser oferecidas aos usuários dos serviços, como o treinamento de todos os profissionais da equipe, a aplicação das medidas em outros cenários, além da unidade de saúde, a inclusão de discussão sobre protocolos de prevenção no âmbito da formação profissional, e um sistema de informação que facilite o registro das medidas preventivas no âmbito da atenção primária.

\section{Referências}

1. Strong K, Mathers C, Leeder S, Beaglehole R. Preventing chronic diseases: how many lives can we save? Lancet. 2005; 366(9496): 1578-82. http:// dx.doi.org/10.1016/S0140-6736(05)67341-2

2. Brasil. Ministério da Saúde. Secretaria de Vigilância em Saúde. Departamento de Analise de Situação de Saúde. Plano de ações estratégicas para o enfrentamento das doenças crônicas não transmissíveis no Brasil 2011-2022. Brasília: Ministério da Saúde; 2011.160 p.

3. Brasil. Ministério da Saúde. A vigilância, o controle e a prevenção das doenças crônicas não transmissíveis: DCNT no contexto do Sistema Único de Saúde brasileiro. Brasília: Organização Pan-Americana da Saúde; 2005. 79 p.

4. Hardy GE Jr. The burden of chronic disease: the future is prevention. Introduction to Dr. James Marks' presentation, "The Burden of Chronic Disease and the Future of Public Health". Prev Chronic Dis. 2004; 1(2): A04.

5. McWhinney IR, Freeman T. Manual de Medicina de Família e Comunidade. 3. ed. Porto Alegre: ArtMed; 2009. p. 956. 
6. Brasil. Ministério da Saúde. Secretaria de Atenção à Saúde. Departamento de Atenção Básica. Rastreamento. Cad Atenção Primária. 2010; (29).

7. Sankarankutty AK, Ribeiro LP. O Exame Periódico no Universo da Atenção Primária. Ribeirão Preto: Hospital das Clínicas da Faculdade de Medicina de Ribeirão Preto da Universidade de São Paulo; 2003. Mimeo.

8. New grades for recommendations from the Canadian Task Force on Preventive Health Care. CMAJ. 2003; 169(3): 207-8.

9. U.S. Preventive Services Task Force [internet]. Rockville: Agency for Healthcare Research and Quality; 2007. Guide to Clinical Preventive Services. [acesso em 12 set 2007]. Disponível em: http://www.ahrq.gov/clinic/prevenix.htm.

10. The Canadian Task Force on Preventive Health Care [internet]. Ontário: The Organization; 2005. [acesso em 12 set 2007]. Disponível em: http:// canadiantaskforce.ca/guidelines/all-guidelines/

11. Programa de Actividades Preventivas y de Promoción de la Salud [página da internet]. Barcelona: Sociedad Española de Medicina de Familia y Comunitaria (semFYC); 2004. [acesso em 12 set 2007]. Disponível em: http://www.papps.org/

12. Campos MAF. Percepção e avaliação dos alunos do curso de medicina de uma escola médica pública sobre a importância do estágio em saúde da família na sua formação. [Dissertação]. Ribeirão Preto: Faculdade de Medicina de Ribeirão Preto, Universidade de São Paulo; 2006.

13. Setz VG, D'Innocenzo M. Avaliação da qualidade dos registros de enfermagem no prontuário por meio da auditoria. Acta Paul Enferm. 2009; $22(3)$ : 313-7. Sistema de Informação da Atenção Básica (SIAB). http://dx.doi.org/10.1590/S0103-21002009000300012

14. Modesto MG, Moreira EC, Almeida-Filho N. Reforma Sanitária e Informação em Saúde: Avaliação dos Registros Médicos em um Distrito Sanitário de Salvador, Bahia. Cad Saúde Pública. 1992; 8(1): 62-68. http://dx.doi.org/10.1590/S0102-311X1992000100008

15. Tonani M, Carvalho EC. Risco de Câncer e Comportamentos preventivos: a persuasão como estratégia de intervenção. Rev Latino-Am Enfermagem. 2008; 16(5): 67-74.

16. Susman J. An ounce of prevention is a ton of work. Fam Med. 2002; 34(1): 45-7.

17. Damiani G, Pinnarelli L, Colosimo SC, Almiento R, Sicuro L, Galasso R, et al. The effectiveness of computerized clinical guidelines in the process of care: a systematic review. BMC Health Serv Res. 2010; 10(1): 2. http://dx.doi.org/10.1186/1472-6963-10-2

18. Starfield B, Hyde J, Gérvas J, Heath I. The concept of prevention: a good idea gone astray? J Epidemiol Community Health. 2008; 62(7): 580-3. http://dx.doi.org/10.1136/jech.2007.071027

19. Instituto Nacional de Câncer. Diagnóstico de câncer Colorretal. [acesso em 06 mar 2013]. Disponível em: http://www2.inca.gov.br/wps/wcm/connect/ tiposdecancer/site/home/colorretal/diagnostico1

20. Santos LL. Análise da percepção e registro das medidas de prevenção de doenças por profissionais da atenção primária. [Tese]. Ribeirão Preto: Faculdade de Medicina de Ribeirão Preto, Universidade de São Paulo; 2010.

21. Rose G. Estratégias da Medicina Preventiva. Norman, AH, tradutor. Porto Alegre: Artmed; 2010. p. 192. 


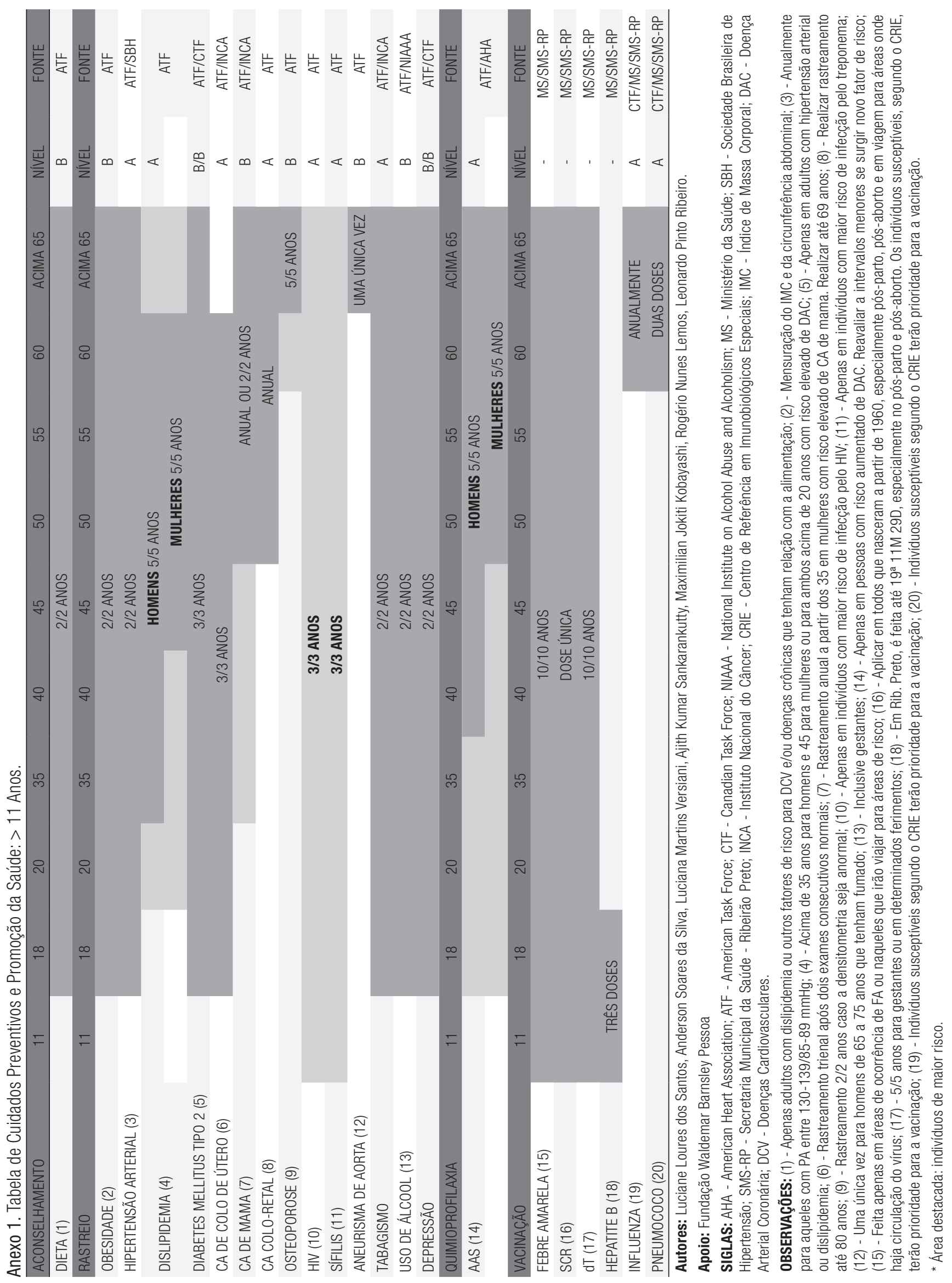



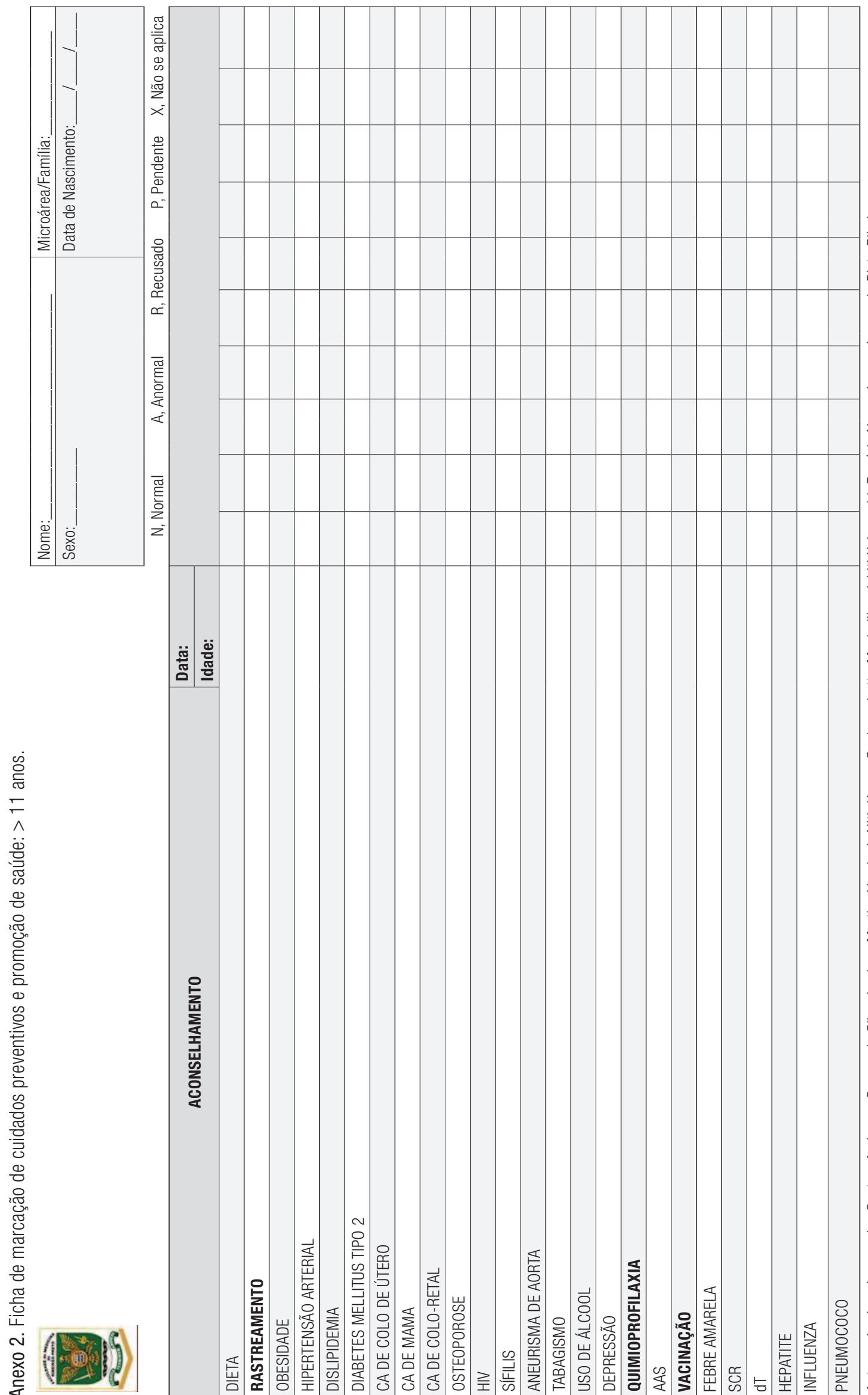

这递

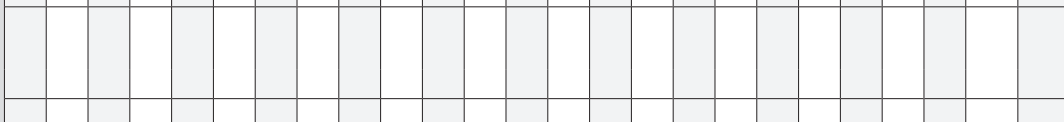

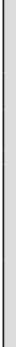

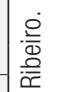

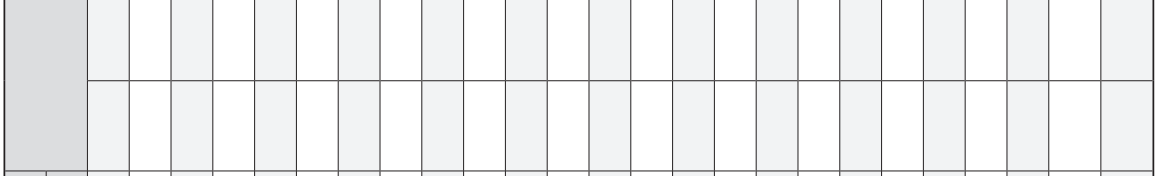

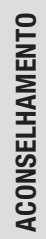

